Check for updates

Cite this: RSC Adv., 2019, 9, 39488

Received 14th August 2019

Accepted 14th November 2019

DOI: $10.1039 / \mathrm{c} 9 \mathrm{ra06365j}$

rsc.li/rsc-advances

\section{Photodegradation of Si-doped GaAs nanowire $\uparrow$}

\author{
A. C. S. Pimenta, (D) ${ }^{* a}$ H. Limborço, ${ }^{b}$ J. C. González, (D) ${ }^{c}$ N. Cifuentes, ${ }^{c}$ \\ Sérgio L. L. M. Ramos ${ }^{d}$ and Franklin M. Matinaga ${ }^{a}$
}

Researching optical effects in nanowires may require a high pump intensity which under ambient conditions can degrade nanowires due to thermal oxidation. In this work we investigated the photodegradation of a single Si-doped GaAs nanowire by laser heating in air. To understand the changes that occurred on the nanowire we carried out Raman spectroscopy, scanning electron microscopy, energy dispersive X-ray spectroscopy, and photoluminescence spectroscopy in laser damaged regions as well as in non-affected ones. From Raman Stokes and anti-Stokes measurements we estimated the local temperature that the oxidation process of the nanowire (NW) surface starts at as $661 \mathrm{~K}$, resulting in two new Raman modes at $200 \mathrm{~cm}^{-1}$ and $259 \mathrm{~cm}^{-1}$. Scanning electron microscopy and energy dispersive $\mathrm{X}$-ray spectroscopy measurements showed a significant loss of arsenic in the oxidized regions, but no erosion of the nanowire. Micro-photoluminescence measurements showed the near-band-edge emission of GaAs along the nanowire, as well as a new emission band at $755 \mathrm{~nm}$ corresponding to polycrystalline $\beta-\mathrm{Ga}_{2} \mathrm{O}_{3}$ formation. Our results also indicate that neither amorphous As nor crystalline As were deposited on the surface of the nanowire. Combining different experimental techniques, this study showed the formation of polycrystalline $\beta-\mathrm{Ga}_{2} \mathrm{O}_{3}$ by oxidation of the nanowire surface and the limits for performing spectroscopic investigations on individual GaAs NWs under ambient air conditions.

\section{Introduction}

In recent years many studies have been devoted to the study of semiconductor nanowires (NWs). ${ }^{1-4}$ These nanostructures have attracted great interest due to their versatile optical ${ }^{2-4}$ and electrical properties, ${ }^{4-6}$ allowing them to be used as a building block for devices ${ }^{4-9}$ or even as an effective platform for an interface with biological systems. ${ }^{10-12}$ Thus, understanding the optical properties of these NWs is fundamental for the development of new technologies. However, some optical measurements require high pump power laser excitation, ${ }^{13}$ which may heat or damage the NW. Only very few works ${ }^{14-18}$ have focused on studying such a problem, where the heating caused by laser irradiation promoted local and irreversible damage to the NW.

In particular, thermal oxidation due to laser heating is an example of photodegradation, in which the oxidation process is accelerated by virtue of the laser incidence in an oxidizing atmosphere. ${ }^{14-16}$ As a consequence of this process, degradation of some features of the sample may occur, such as a change in the chemical composition due to native oxide formation; ${ }^{19}$

${ }^{a}$ Photonics Laboratory, Physics Department, UFMG, Belo Horizonte, Brazil. E-mail: matinaga@fisica.ufmg.br

${ }^{b}$ Microscopy Centre of UFMG, UFMG, Belo Horizonte, Brazil

${ }^{c}$ Nanodevices and Sensors Laboratory, UFMG, Belo Horizonte, Brazil

${ }^{d}$ Centre for Nanomaterial Technology and Graphene - CTNano, Belo Horizonte, Brazil

† Electronic supplementary information (ESI) available. See DOI: 10.1039/c9ra06365j morphological modifications ${ }^{16}$ as in the case of surface erosion caused by loss of sample constituents; ${ }^{14}$ or even suppression of optical emission efficiency. ${ }^{15}$ Thermal oxidation in bulk GaAs has been extensively reported in the literature,,$^{15,19-32}$ and all authors agree on the formation of gallium oxides. Recently, Yazji et al. ${ }^{15}$ studied the local modifications suffered by a GaAs NW after a laser irradiation induced thermal oxidation process in air for local temperatures in the 500-600 K range. They observed that the photoluminescence emission was suppressed in the photodegraded region, but in contrast they reported a local increase of the thermal resistance of the sample. This latter conclusion was justified by the appearance of amorphous As (a-As) at low local temperatures, followed by the formation of crystalline arsenic (c-As) (i.e. aggregates of arsenic atoms with some periodic arrangement) as the local temperature increased. They claim that a-As and c-As were formed as thermal oxidation by-products. That work is based on a previous work by Campbell et al., ${ }^{33}$ in which the photodegradation of bulk GaAs caused by laser irradiation was studied by Raman and photoluminescence spectroscopy. In the latter study, the appearance of a broad Raman band at $190-260 \mathrm{~cm}^{-1}$ for temperatures up to $500 \mathrm{~K}$ was attributed to the formation of a-As. By increasing the laser power density and consequently the local temperature of the sample up to $1300 \mathrm{~K}$, they observed the appearance of two new Raman modes at $190 \mathrm{~cm}^{-1}$ and $250 \mathrm{~cm}^{-1}$, attributed to the formation of c-As. Note the strong temperature difference in the appearance of the c-As Raman peaks between both studies. Theoretical works have also shown the possibility of c-As 
formation during the thermal oxidation process, ${ }^{30}$ however, beyond certain temperatures its evaporation may occur, which is expected for bulk III-V semiconductor compounds. ${ }^{\mathbf{3 4}}$ For example, He and coworkers ${ }^{14}$ reported c-As formation in an InAs NW, when it was irradiated by a laser. They observed the evaporation of As from the NW with increasing laser power, inducing ablation and consequently fracture of the NW. Thus, although there is a consensus about native oxide formation as by-products, ${ }^{35}$ some aspects related to the oxidation of GaAs NWs in air, the formation of a-As, c-As and their accumulation on the GaAs NW surface during laser heating need further investigation.

In order to comprehend the photodegradation of GaAs NWs, we investigated their thermal oxidation process in air induced by laser heating in a broad local temperature range. As will be shown, the increase of the laser beam power promoted heating of a single NW leading to local oxidation above $661 \mathrm{~K}\left(388{ }^{\circ} \mathrm{C}\right)$. Scanning electron microscopy (SEM) and energy-dispersive Xray spectroscopy (EDS) were used to investigate morphological and chemical changes in photodegraded regions of the NW. Moreover, we examined by micro-Raman ( $\mu$-RS) and microphotoluminescence ( $\mu-\mathrm{PL})$ spectroscopic mapping two regions of the NW at different stages of oxidation. Based on our studies of the structural, morphological, chemical and electronic properties of the NW we show that the NW suffered photodegradation caused by a large reduction of its arsenic content and by gallium oxide formation. However, indications of the formation of amorphous or even crystalline As as oxidation byproducts were not found.

\section{Experimental details}

Free standing Si-doped GaAs nanowires (NWs) were grown on a silicon substrate by molecular beam epitaxy in a Riber 2300 R\&D system, ${ }^{2,36}$ by the vapor-liquid-solid mechanism assisted by Au-nanoparticles. The nominal silicon concentration was 1.0 $\times 10^{16} \mathrm{~cm}^{-3}$. To study a single NW, the NWs were mechanically removed and transferred onto another Si substrate coated with a $300 \mathrm{~nm}$ thick layer of $\mathrm{SiO}_{2}$. These GaAs nanowires exhibit polytypism, which is the coexistence of alternating segments of zinc blende (ZB) and wurtzite (WZ) phases along the NW axis. ${ }^{2,4,13,36,37}$

In order to investigate the thermal oxidation process, we carried out $\mu$-RS in backscattering geometry by using a WITec confocal Raman spectroscopy system equipped with both piezoelectric and motorized scanning stages. These measurements were executed at three nearby spots on the NW, separated by approximately $700 \mathrm{~nm}$, at room temperature and in contact with air. A $532 \mathrm{~nm}$ laser line (continuous wave - CW) was focused on the sample with a $100 \times$ objective lens with a numerical aperture of 0.90 . Considering Porto's notation, ${ }^{38}$ the incoming light $\varepsilon_{\mathrm{i}}$ was polarized parallel to the growth axis of the NW ([0001] for WZ segments and [111] for ZB ones) and the polarization of the scattered light $\varepsilon_{\mathrm{s}}$ was not analyzed. Stokes and anti-Stokes measurements were performed with accumulation of 10 minutes and increasing the pump power in discrete steps.
Following this characterization, the morphological and chemical composition of the NW were studied by SEM and EDS. These measurements were executed in a FEI Dual Beam Quanta $3 \mathrm{D}$ FEG system at $20 \mathrm{kV}$ high voltage. EDS and SEM were carried out in the photodegraded areas as well as in the non-degraded ones.

In sequence, a fourth nearby ( $5 \mu \mathrm{m}$ away) spot on the NW was oxidized under the same conditions, thereby providing two different regions on the NW with different stages of oxidation. The second region (fourth spot) was just oxidized, while the first region (first three spots) was oxidized and subsequently annealed at a lower temperature (by conduction) during the oxidation of the second region. For a more complete characterization we acquired low-pump power $\mu$-RS maps of nonoxidized and both thermally oxidized regions of the NW. Moreover, to investigate the optical emission properties of the NW and of the thermal oxidation by-products we performed low-temperature $\mu$-PL measurements using a Linkan THMSE600 liquid nitrogen cryostat to stabilize the temperature at $90 \mathrm{~K}$. The same WITec system was used for $\mu$-PL mapping, but by using a $475 \mathrm{~nm}$ laser line and a $50 \times$ objective lens (NA 0.55).

\section{Results and discussion}

The evolution of the Raman spectrum as a function of pump power density is presented in the map of Fig. 1(a). This map is composed only of Stokes peaks, and their intensities were normalized by the maximum for each spectrum. At low pump power density we can see the longitudinal optical $\nu_{\mathrm{LO}, \mathrm{ZB}}=$ $291 \mathrm{~cm}^{-1}$ and transverse optical $\nu_{\mathrm{TO}, \mathrm{ZB}}=267 \mathrm{~cm}^{-1}$ modes of the ZB phase as well as the $\nu_{\mathrm{TO}, \mathrm{WZ}}=267 \mathrm{~cm}^{-1}\left(\mathrm{E}_{1}^{\mathrm{H}}\right)$ mode of the WZ phase, see Fig. 1(b). However, as the pump power density increases the peaks shift due to the local heating of the NW. Above $184 \mathrm{~kW} \mathrm{~cm}{ }^{-2}$, two new peaks appear around $200 \mathrm{~cm}^{-1}$ and $259 \mathrm{~cm}^{-1}$ (Fig. 2 of ESI $\dagger$ ). The arising of these new modes reveals an irreversible change in the NW nature, since these modes are related to the photodegradation process (Fig. 1 of ESI $\dagger$ ). These new modes were firstly reported in 1977 by Cape et al. after the thermal oxidation of bulk GaAs, ${ }^{29}$ nevertheless, they did not identify the origin of these peaks. Based on the literature and considering the reported chemical reactions for the thermal oxidation of $\mathrm{GaAs},{ }^{30}$ the additional Raman modes could be either associated to the $A_{3 \mathrm{~g}}\left(199 \mathrm{~cm}^{-1}\right)$ mode of $\mathrm{Ga}_{2} \mathrm{O}_{3}$ (ref. 39 and 40) and the $A_{1}\left(260 \mathrm{~cm}^{-1}\right)$ mode of $\mathrm{GaAsO}_{4}$ (ref. 41) or to the $\mathrm{TO}_{\mathrm{As}}\left(198 \mathrm{~cm}^{-1}\right)$ and $\mathrm{LO}_{\mathrm{As}}\left(257 \mathrm{~cm}^{-1}\right)$ modes of c-As. ${ }^{31}$ There is a consensus about gallium oxide formation, ${ }^{26,30,32,42}$ however, some authors ${ }^{15,31,33}$ assign these additional Raman modes to crystalline arsenic (c-As).

Analyzing the Raman map of Fig. 1(a) again, it is possible to observe that the relative intensity of the oxide peaks increases significantly with the pump power density. The positions of these two peaks also downshift with the pump power density. These effects are clearly shown in Fig. 1(c), associated with the highest value of pump power density, corresponding to 1092 $\mathrm{kW} \mathrm{cm}^{-2}$. In this spectrum, besides the GaAs modes and the oxide ones $\left(200 \mathrm{~cm}^{-1}\right.$ and $\left.259 \mathrm{~cm}^{-1}\right)$, we can see a silicon mode from the substrate at approximately $300 \mathrm{~cm}^{-1}$, and another 


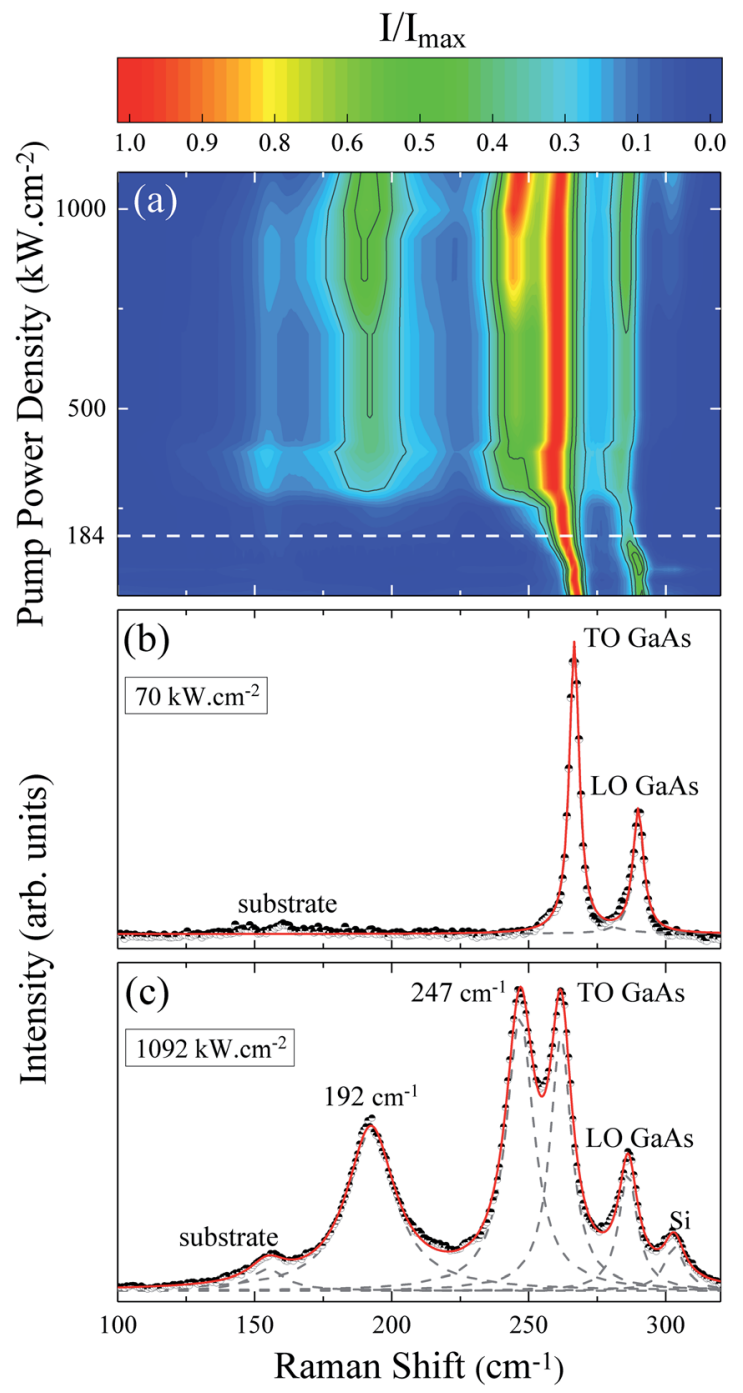

Fig. 1 (a) Raman scattering intensities as a function of pump power density. The dashed line represents the threshold pump power density for the emergence of oxide peaks. (b) Spectrum taken at $L=70 \mathrm{~kW}$ $\mathrm{cm}^{-2}$. (c) Spectrum taken at $L=1092 \mathrm{~kW} \mathrm{~cm}{ }^{-2}$. The dots are the experimental measurements, while the dashed Lorentzians represent fitting of the individual mode contributions and the red line is the total fitting curve.

substrate peak at $154 \mathrm{~cm}^{-1}$. These last two modes were verified through measurements done on clean substrate areas and they are not detected for low pump power conditions. In the Raman scattering theory the peak intensities are calculated from the differential spectral cross section, which in turn is related to the amount of sample which contributes to the detected scattered light. ${ }^{43}$ Hence, the increase in normalized intensity of the oxide peaks suggests that related by-products are being formed continuously as the pump power density increases and are accumulating on the GaAs NW. Note that the whole spectrum is well fitted by a combination of Lorentzian lines centered at the position of the above mentioned modes. An a-As mode ${ }^{15,33}$ at $220 \mathrm{~cm}^{-1}$ was not necessary. The ascription of a $220 \mathrm{~cm}^{-1}$ mode to a-As was previously attributed to the formation of c-As during the oxidation of GaAs by laser heating. ${ }^{15,33}$

The local temperature $(T)$ of the NW was calculated, as a function of pump power density $(L)$, by using the ratio between the Stokes $\left(I_{\mathrm{S}}\right)$ and anti-Stokes $\left(I_{\mathrm{aS}}\right)$ intensities of the GaAs TO mode: ${ }^{44}$

$$
\frac{I_{\mathrm{S}}}{I_{\mathrm{aS}}}=\left(\frac{\omega_{\mathrm{i}}-\omega}{\omega_{\mathrm{i}}+\omega}\right)^{3} \mathrm{e}^{\hbar \omega /\left(k_{\mathrm{B}} T\right)}
$$

where $\omega_{\mathrm{i}}$ represents the laser frequency, $\omega$ is the frequency of the TO GaAs mode and $k_{\mathrm{B}}$ is Boltzmann's constant.

The pump power dependence of the local temperature, as well as the downshift of the GaAs NW TO mode with temperature are shown in Fig. 2. Since the above relation is no longer valid after oxidation, ${ }^{43}$ we only used the data prior to this process, below $L=184 \mathrm{~kW} \mathrm{~cm}{ }^{-2}$. Thus, this value of $L$ represents a threshold temperature $T_{\text {limit }}=661 \mathrm{~K}\left(388^{\circ} \mathrm{C}\right)$ for the start of the oxidation process. This value of the photodegradation temperature is close to the value between 500-600 $\mathrm{K}$ reported by Yazji et $a{ }^{15}$ and the one reported by Persson
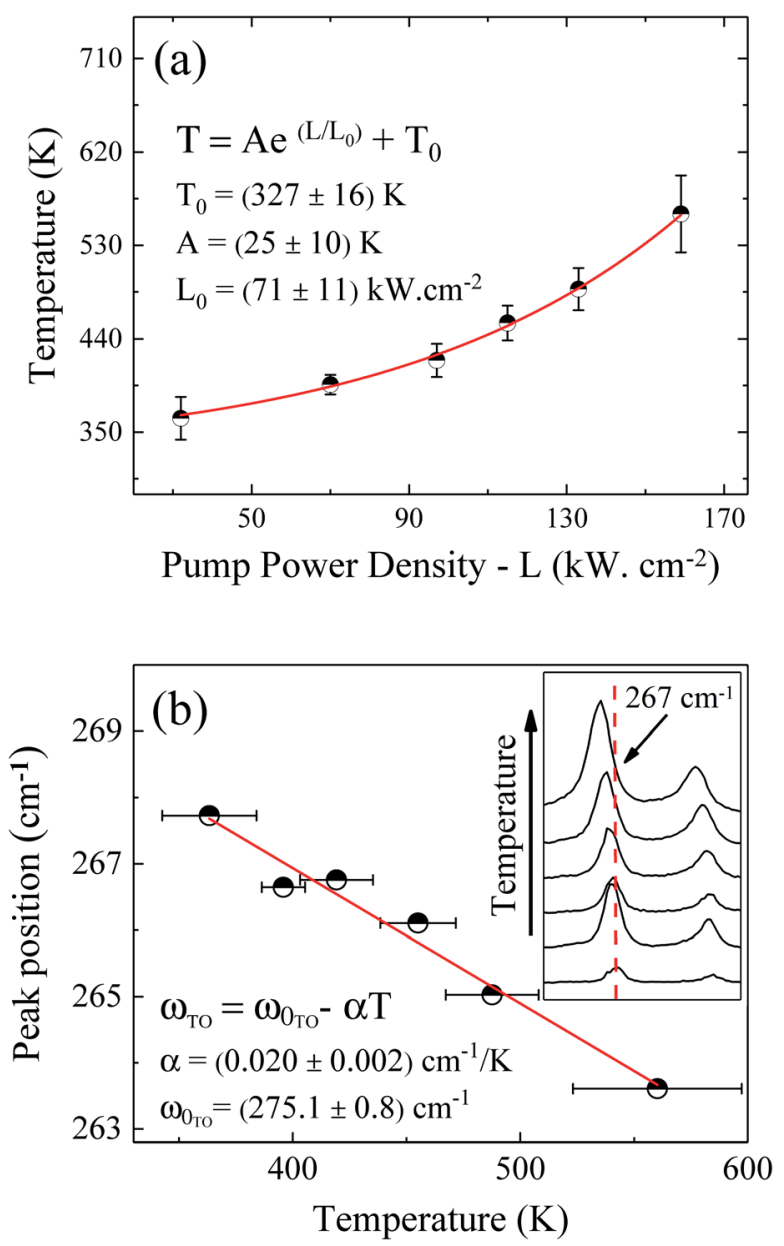

Fig. 2 (a) Pump power density dependence of the GaAs NW local temperature. (b) Temperature dependence of the GaAs TO peak position. The inset of (b) shows explicitly the downshift of the Stokes peak. 
et al., ${ }^{45}$ who observed that the NWs suffered continuous decomposition for temperatures above $673 \mathrm{~K}\left(400{ }^{\circ} \mathrm{C}\right)$.

Laser radiation is the main factor responsible for raising the local temperature. The dependence of the local NW temperature on $L$ is related to the thermal conductivity of GaAs as well as to the fraction of absorbed energy released as heat by the NW; furthermore, other conditions such as light penetration depth and width of the focused beam should also be considered. ${ }^{44}$ In addition to laser incidence, the contact with the environment and the poor heat dissipation between the NW and the Si substrate also contribute to the rise in local temperature and consequently, to the oxidation process. The thermal effects caused by the laser beam also promote the shift of Raman modes, which can be roughly explained by the thermal expansion of the crystal lattice. ${ }^{46}$ Analyzing graph 2(b) which shows the Raman temperature as a function of the GaAs TO mode $\left(\nu_{\mathrm{LO}, \mathrm{GaAs}}=267 \mathrm{~cm}^{-1}\right)$, we can explore the customary linear behavior between the increase of temperature and downshift of the peak, $\omega=\omega_{0}-\alpha T$. From a linear fit we can extract the temperature coefficient $\alpha$, whose value of $0.020 \mathrm{~cm}^{-1} \mathrm{~K}^{-1}$ is in agreement with the one reported for bulk GaAs $0.016 \mathrm{~cm}^{-1}$ $\left.\mathrm{K}^{-1}\right) .{ }^{47}$ Thus, we can ensure that the local temperatures obtained from this method reproduce reliable values.

In accordance with the $\mathrm{Ga}-\mathrm{As}-\mathrm{O}$ equilibrium phase diagram, ${ }^{30}$ some reactions can occur depending on the oxidation conditions, i.e. the temperature and surroundings in which the process takes place. In our experiment, when the process starts, we suppose that the system is in the "weak" oxidizing condition where the main product would be native gallium oxide $\left(\mathrm{Ga}_{2} \mathrm{O}_{3}\right) .{ }^{30}$ For temperatures up to $673 \mathrm{~K}\left(400{ }^{\circ} \mathrm{C}\right)$, gallium oxide is in the amorphous phase. However, its stable polycrystalline phase $\left(\beta-\mathrm{Ga}_{2} \mathrm{O}_{3}\right)$ is reached in the range of $773-973 \mathrm{~K}$ (500-700 $\left.{ }^{\circ} \mathrm{C}\right){ }^{24}$ Arsenic oxide formation $\left(\mathrm{As}_{2} \mathrm{O}_{3}\right)$ could be also expected to occur temporally during the oxidation process, since it is volatile above $623 \mathrm{~K}\left(350{ }^{\circ} \mathrm{C}\right) .{ }^{26,35}$

Throughout our experiments the temperature was not kept constant. Ours is a dynamical process in which other compounds could also be produced. Then, for temperatures above $773 \mathrm{~K}\left(500{ }^{\circ} \mathrm{C}\right)$, it may be possible to detect $\mathrm{GaAsO}_{4}$, due to the fact that some As atoms can react with $\mathrm{Ga}_{2} \mathrm{O}_{3}$ in these conditions. ${ }^{19,24,26}$ Thus, as the pump power increases and, consequently, the temperature too, we would probably achieve "intermediate" oxidizing conditions or even "stronger" ones. ${ }^{30}$ However, only two oxide-related peaks were observed during the whole process. These peaks were observed for temperatures above $661 \mathrm{~K}\left(388{ }^{\circ} \mathrm{C}\right)$, for which arsenic oxides are already volatile. This is a clear indication that new oxide species or a-As were not formed after the initial species at $661 \mathrm{~K}$.

Fig. 3 displays As, Ga, Au and Si EDS elemental maps for the first oxidized region of the NW, including a SEM image of the same region. The EDS maps were obtained from As-L $\alpha$, Ga-L $\alpha$, $\mathrm{Au}-\mathrm{L} \alpha$, and Si-K $\alpha$ X-Ray emission lines. The SEM image (the topmost image in Fig. 3) shows the gold contact on the left side of the NW. It is possible to see a strong contrast between the pristine regions and the oxidized ones. The three oxidized spots (dark spots marked by a white + ) have diameters of about $500 \mathrm{~nm}$, which corresponds to the laser spot size. These contrast

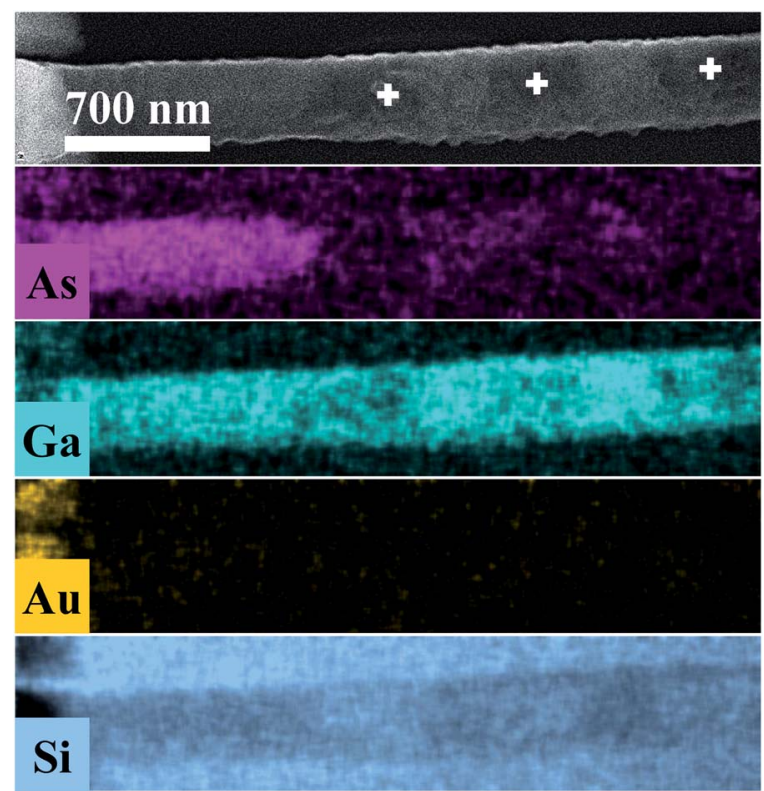

Fig. 3 As, Ga, Au and Si EDS elemental maps for the oxidized regions of the NW. In the uppermost SEM image of the NW, the three markings show the photodegraded areas.

variations in the SEM image are strong evidences that the sample suffered degradation, although we did not observe any decreasing of the diameter or erosion of the NW, as has been described by some researchers in similar experiments. ${ }^{\mathbf{1 4 1 1 5}} \mathrm{A}$ slight decrease of the Ga peak counts, as well as a strong decrease of the As peak counts, can be observed in the EDS maps of the irradiated regions. A semi-quantitative analysis of the Ga and As atomic concentrations along the NW is shown in Fig. 3 and 4 of the ESI. $\uparrow$ From these analyses we observed a $20 \%$ reduction in the amount of As in the irradiated regions. The qualitative analysis of the oxygen concentration, shown in Fig. 3 and 4 of ESI, $\uparrow$ also demonstrates a significant increase of oxygen in the same regions. According to the literature in "weak" oxidizing conditions, ${ }^{30}$ production of crystalline arsenic is expected in this process. However, rich regions of As (corresponding to a-As or even c-As agglomerations) were not found in our measurements. This observation is in agreement with the lack of observation of the a-As Raman mode, and indicates the fast evaporation of As and related oxides during the heating process.

The oxidation of the second region promoted different stages of oxidation in the NW, since the first region was annealed (by conduction) during the oxidation of the second one. These two different stages were investigated and verified by means of $\mu$-RS and $\mu$-PL mappings along the NW. Fig. 4 shows low-pump Raman maps of the first and second regions of the NW. A deconvolution analysis was applied to retrieve the main spectral components of the Raman maps, constituting the entire Raman hyperspectral. This procedure resulted in three different spectra presented in Fig. 4. The first spectrum (blue) reproduces the non-oxidized region, where we have only GaAs modes, as expected. From its corresponding map (on the right 


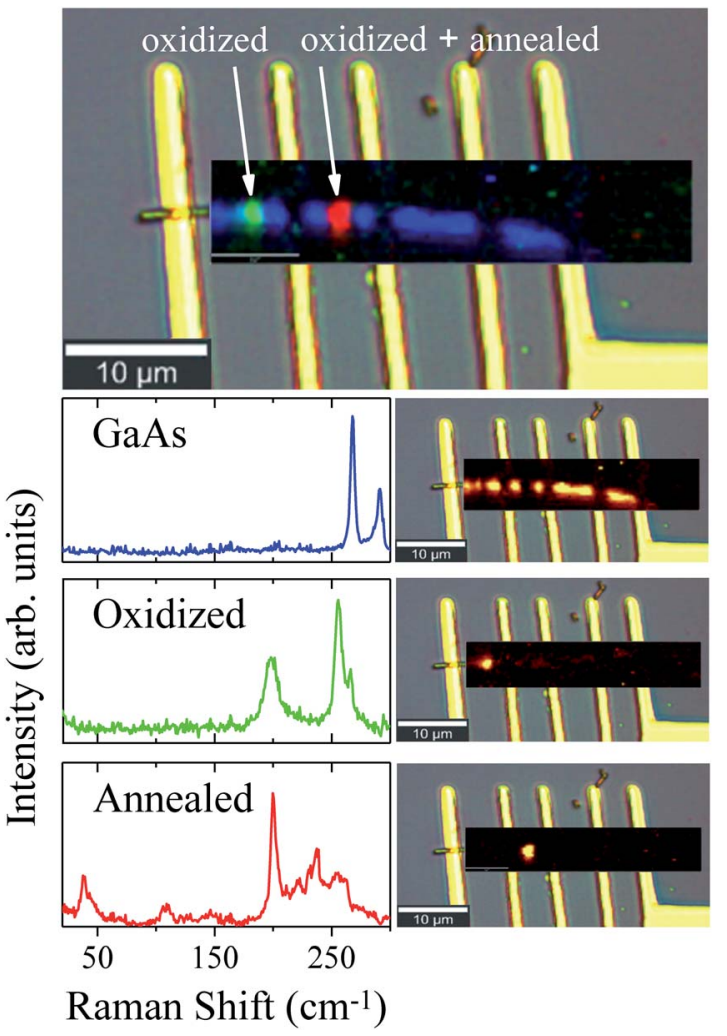

Fig. 4 Combined spectral component distribution map of the Raman hyperspectral (topmost image) and, in sequence, the separate distributions maps, together with their respective spectra (left side) for each component after the deconvolution analysis.

side), which shows how this spectral component is spatially distributed upon spectral deconvolution, we observe that an intense signal is present for most of the NW extension. However, in the photodegraded areas the GaAs modes are observed to be strongly reduced. The green and red Raman spectra represent the two differently oxidized regions. Examining the green spectrum, of the second oxidized region, we observe peaks at $200 \mathrm{~cm}^{-1}$ and $259 \mathrm{~cm}^{-1}$ which are associated to expected oxidation products. The corresponding map (on the right side) shows this component in a specific region of the NW. Differently, the red spectrum of the first region (oxidized and also annealed) shows low-frequency Raman modes at $39 \mathrm{~cm}^{-1}$ and $109 \mathrm{~cm}^{-1}$, besides those mentioned above, indicating some modification of the initial oxides. Since this region was heated again after oxidation, it is expected that the sample is in another stage of oxidation - it is in a higher degradation state.

To further understand the photodegradation suffered by the $\mathrm{NW}, \mu$-PL mappings were measured at $90 \mathrm{~K}$. We obtained two different spectral components from the deconvolution procedure, as can be observed in Fig. 5. The blue spectrum depicts near-band-edge GaAs photoluminescence with a maximum around $830 \mathrm{~nm}$. The combined component distribution map (on the right side of the spectrum) shows GaAs emission along the whole NW, less intense in the oxidized areas. PL peaks associated to $\mathrm{Ga}_{2} \mathrm{O}_{3}$ and $\mathrm{GaAsO}_{4}$ would not be observed in oxidized regions, since the bandgaps of these compounds ${ }^{48,49}$

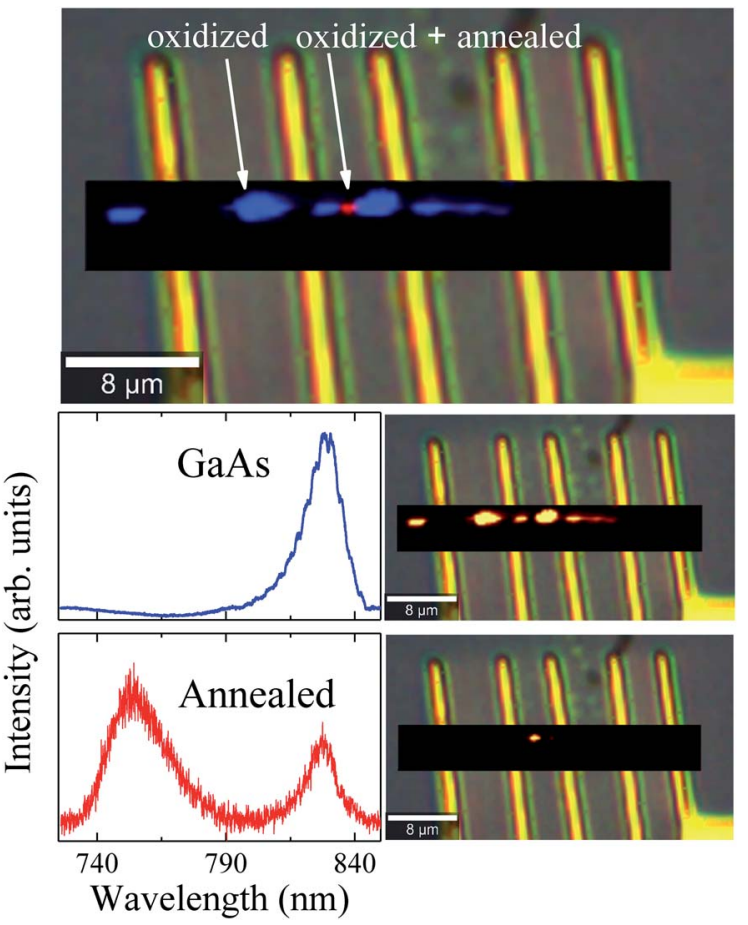

Fig. 5 Combined spectral component distribution map for the $\mu$-PL hyperspectral (topmost image) and, in sequence, the individual maps, together with their respective spectra (on the left side), for each spectral component after the deconvolution analysis.

are too large to be excited with the $475 \mathrm{~nm}$ laser line used in our measurements. On the other hand, a strong PL emission at $755 \mathrm{~nm}$ was observed in the annealed region of the NW (red spot, Fig. 5). Cheng et al. ${ }^{50}$ have observed that two new emissions at $330 \mathrm{~nm}$ and $706 \mathrm{~nm}$ appeared when the $\beta-\mathrm{Ga}_{2} \mathrm{O}_{3}$ was annealed, associated to defect levels transitions, generated during this process. Under these considerations, we suggest that the gallium oxide amorphous phase was formed above $T_{\text {limit }}$ and transformed to the $\beta-\mathrm{Ga}_{2} \mathrm{O}_{3}$ polycrystalline phase as the local temperature increased. Finally, during the annealing of the already oxidized region, defect levels were generated within the $\beta-\mathrm{Ga}_{2} \mathrm{O}_{3}$ bandgap, producing the emission observed at $755 \mathrm{~nm}$. Therefore, the $\mu$-PL measurements support the formation of this kind of gallium oxide. Moreover, this outcome is evidence that the region achieved a local temperature above $773 \mathrm{~K}\left(500^{\circ} \mathrm{C}\right)$, which is similar to the formation temperature of $\beta-\mathrm{Ga}_{2} \mathrm{O}_{3}{ }^{51}$ Finnie et al. ${ }^{52}$ have shown that microcrystalline arsenic oxides, as well as bulk $\mathrm{As}_{2} \mathrm{O}_{3}$ and $\mathrm{As}_{2} \mathrm{O}_{5}$, exhibit strong PL emission at $548 \mathrm{~nm}$. However, emission at this wavelength was not observed in our case. This last result is also in agreement with the $\mu$-RS and EDS measurements, where evidences of amorphous arsenic, c-As or arsenic oxides were not observed.

\section{Conclusions}

In summary it has been shown that under ambient conditions GaAs NWs suffer photodegradation for a laser pump power density above $184 \mathrm{~kW} \mathrm{~cm}{ }^{-2}$, which corresponds to a local 
temperature above $661 \mathrm{~K}\left(388^{\circ} \mathrm{C}\right)$. Furthermore, above this temperature we verified two new modes at $200 \mathrm{~cm}^{-1}$ and $259 \mathrm{~cm}^{-1}$ in the Raman spectra, which were related to the thermal oxidation process. Some researchers ${ }^{15,31,33}$ have attributed these two Raman modes to crystalline arsenic. However, we verified the absence of amorphous As Raman modes at $220 \mathrm{~cm}^{-1}$, the large reduction of the amount of As in the laser irradiated regions, and the absence of the characteristic green PL emission of arsenic oxides at $548 \mathrm{~nm}$ in our experiments. Thus, our results indicate that neither amorphous As, nor crystalline As, were deposited and oxidized on the surface of the GaAs NW during the laser heating and oxidation process. Furthermore, our measurements provide strong evidence for gallium oxide formation in its $\beta$-phase. Therefore, we ascribe the observed additional Raman modes at $200 \mathrm{~cm}^{-1}$ to this oxide. We conclude that the photodegradation of GaAs NWs by strong laser irradiation (up to $1092 \mathrm{~kW} \mathrm{~cm}^{-2}$ ) does not affect significantly the morphology of the NW, but results in polycrystalline gallium oxide formation, severe arsenic loss and reduction of the near-band-edge photoluminescence emission only in the oxidized and further annealed regions. Lastly, by combining different experimental techniques, our study clearly shows the limits under which spectroscopic investigations on individual GaAs NWs can be performed under ambient air conditions in terms of laser power density and local temperature.

\section{Conflicts of interest}

There are no conflicts of interest to declare.

\section{Acknowledgements}

This work was funded by the Brazilian agencies CNPq, FAPEMIG and CAPES.

\section{Notes and references}

1 J. Lu, H. Liu, X. Zhang and C. H. Sow, Nanoscale, 2018, 10, 17456-17476.

2 N. B. Sedrine, R. Ribeiro-Andrade, A. Gustafsson, M. Soares, J. Bourgard, J. Teixeira, P. Salomé, M. Correia, M. Moreira, A. De Oliveira, et al., Nanoscale, 2018, 10, 3697-3708.

3 B. Falcão, J. Leitão, M. Correia, M. Soares, F. Morales, J. Mánuel, R. Garcia, A. Gustafsson, M. Moreira, A. De Oliveira, et al., J. Appl. Phys., 2013, 114, 183508.

4 P. Kannappan, N. B. Sedrine, J. P. Teixeira, M. R. Soares, B. P. Falcão, M. R. Correia, N. Cifuentes, E. R. Viana, M. V. Moreira, G. M. Ribeiro, et al., Beilstein J. Nanotechnol., 2017, 8, 2126-2138.

5 N. Cifuentes, H. Limborço, E. Viana, D. Roa, A. Abelenda, M. da Silva, M. Moreira, G. Ribeiro, A. de Oliveira and J. C. González, Phys. Status Solidi B, 2016, 253, 1960-1964.

6 N. Cifuentes, E. Viana, H. Limborço, D. Roa, A. Abelenda, M. da Silva, M. Moreira, G. Ribeiro, A. de Oliveira and J. C. González, J. Nanomater., 2016, 2016, 9454319.

7 J. B. Baxter and E. S. Aydil, Appl. Phys. Lett., 2005, 86, 053114.
8 B. Hua, Q. Lin, Q. Zhang and Z. Fan, Nanoscale, 2013, 5, 6627-6640.

9 J. Yan, Y. Chen, X. Wang, Y. Fu, J. Wang, J. Sun, G. Dai, S. Tao and Y. Gao, Nanoscale, 2019, 11, 2162-2169.

10 Y. Cui, Q. Wei, H. Park and C. M. Lieber, Science, 2001, 293, 1289-1292.

11 S. Wang, L.-P. Xu, H.-W. Liang, S.-H. Yu, Y. Wen, S. Wang and X. Zhang, Nanoscale, 2015, 7, 11460-11467.

12 S. Su, Y. He, S. Song, D. Li, L. Wang, C. Fan and S.-T. Lee, Nanoscale, 2010, 2, 1704-1707.

13 A. C. S. Pimenta, D. Teles Ferreira, D. Roa, M. Moreira, A. De Oliveira, J. C. González, M. De Giorgi, D. Sanvitto and F. M. Matinaga, J. Phys. Chem. C, 2016, 120, 17046-17051.

14 J. He, P. Chen, W. Lu, N. Dai and D.-M. Zhu, Appl. Phys. A: Mater. Sci. Process., 2014, 115, 885-893.

15 S. Yazji, I. Zardo, M. Soini, P. Postorino, A. F. i. Morral and G. Abstreiter, Nanotechnology, 2011, 22, 325701.

16 V. K. Gupta, A. A. Ingale, V. Jain, R. Aggarwal and S. Pal, J. Alloys Compd., 2018, 735, 1331-1338.

17 R. Tanta, M. Madsen, Z. Liao, P. Krogstrup, T. Vosch, J. Nygård and T. S. Jespersen, Appl. Phys. Lett., 2015, 107, 243101.

18 R. Tanta, T. Kanne, F. Amaduzzi, Z. Liao, M. H. Madsen, E. Alarcón-Lladó, P. Krogstrup, E. Johnson, A. F. i. Morral, T. Vosch, et al., Nanotechnology, 2016, 27, 305704.

19 C. Wilmsen, J. Vac. Sci. Technol., 1981, 19, 279-289.

20 S. Murarka, Appl. Phys. Lett., 1975, 26, 180-181.

21 M. Rubenstein, J. Electrochem. Soc., 1966, 113, 540-542.

22 H. T. Minden, J. Electrochem. Soc., 1962, 109, 733.

23 A. Pakes, P. Skeldon, G. Thompson, R. Hussey, S. Moisa, G. Sproule, D. Landheer and M. Graham, Surf. Interface Anal., 2002, 34, 481-484.

24 O. R. Monteiro and J. W. Evans, J. Vac. Sci. Technol., A, 1989, 7, 49-54.

25 G. Schwartz, G. Gualtieri, J. Griffiths, C. Thurmond and B. Schwartz, J. Electrochem. Soc., 1980, 127, 2488-2499.

26 G. Hollinger, R. Skheyta-Kabbani and M. Gendry, Phys. Rev. B: Condens. Matter Mater. Phys., 1994, 49, 11159.

27 N. Levinsohn, R. Beserman, C. Cytermann, R. Brener, Y. L. Khait, G. Regel, J. Musolf, M. Weyers, A. Brauers and P. Balk, Appl. Phys. Lett., 1990, 56, 1131-1133.

28 F. Koshiga and T. Sugano, Jpn. J. Appl. Phys., 1977, 16, 465. 29 J. Cape, W. Tennant and L. Hale, J. Vac. Sci. Technol., 1977, 14, 921-923.

30 C. Thurmond, G. Schwartz, G. Kammlott and B. Schwartz, J. Electrochem. Soc., 1980, 127, 1366-1371.

31 R. L. Farrow, R. K. Chang, S. Mroczkowski and F. H. Pollak, Appl. Phys. Lett., 1977, 31, 768-770.

32 T. Sands, J. Washburn and R. Gronsky, Mater. Lett., 1985, 3, 247-250.

33 I. Campbell and P. Fauchet, Appl. Phys. Lett., 1990, 57, 10-12. 34 S. J. Pearton, C. R. Abernathy and F. Ren, Topics in growth and device processing of III-V semiconductors, World scientific, 1996, vol. 1.

35 J. Contour, J. Massies, H. Fronius and K. Ploog, Jpn. J. Appl. Phys., 1988, 27, L167. 
36 B. Falcão, J. Leitão, J. C. González, M. Correia, K. ZayasBazán, F. M. Matinaga, M. Moreira, C. Leite and A. De Oliveira, J. Mater. Sci., 2013, 48, 1794-1798.

37 B. P. Falcao, J. P. Leitão, M. R. Correia, M. F. Leitão, M. R. Soares, M. V. Moreira, A. G. de Oliveira, F. M. Matinaga and J. C. González, J. Mater. Chem. C, 2014, 2, 7104-7110.

38 I. Zardo, S. Conesa-Boj, F. Peiro, J. Morante, J. Arbiol, E. Uccelli, G. Abstreiter and A. F. i. Morral, Phys. Rev. B: Condens. Matter Mater. Phys., 2009, 80, 245324.

39 C. Kranert, C. Sturm, R. Schmidt-Grund and M. Grundmann, Sci. Rep., 2016, 6, 35964.

40 S. Pearton, J. Yang, P. H. Cary IV, F. Ren, J. Kim, M. J. Tadjer and M. A. Mastro, Appl. Phys. Rev., 2018, 5, 011301.

41 O. Cambon, G. Bhalerao, D. Bourgogne, J. Haines, P. Hermet, D. Keen and M. Tucker, J. Am. Chem. Soc., 2011, 133, 8048-8056.

42 C. Grovenor and A. Cerezo, J. Appl. Phys., 1989, 65, 50895095.

$43 \mathrm{~W}$. Hayes and R. Loudon, Scattering of light by crystals, Courier Corporation, 2012.
44 M. J. Pelletier, Analytical applications of Raman spectroscopy, Wiley-Blackwell, 1999.

45 A. I. Persson, M. W. Larsson, S. Stenström, B. J. Ohlsson, L. Samuelson and L. R. Wallenberg, Nat. Mater., 2004, 3, 677.

46 L. Han, M. Zeman and A. H. Smets, Nanoscale, 2015, 7, 83898397.

47 J. Besson, J. Itie, A. Polian, G. Weill, J. Mansot and J. Gonzalez, Phys. Rev. B: Condens. Matter Mater. Phys., 1991, 44, 4214.

48 L. Li, E. Auer, M. Liao, X. Fang, T. Zhai, U. K. Gautam, A. Lugstein, Y. Koide, Y. Bando and D. Golberg, Nanoscale, 2011, 3, 1120-1126.

49 D. M. Christie and J. R. Chelikowsky, J. Phys. Chem. Solids, 1998, 59, 617-624.

50 Y. Cheng, J. Chen, K. Yang, Y. Wang, Y. Yin, H. Liang and G. Du, J. Vac. Sci. Technol., B, 2014, 32, 03 D119.

51 S. Stepanov, V. Nikolaev, V. Bougrov and A. Romanov, Rev. Adv. Mater. Sci., 2016, 44, 63-86.

52 C. M. Finnie and P. W. Bohn, Appl. Phys. Lett., 1999, 74, 1096-1098. 